\title{
Comparison of Quantitative Electroencephalography between Tic Disorder and Attention-Deficit/Hyperactivity Disorder in Children
}

\author{
Ilju Lee', Jiryun Lee ${ }^{2}$, Myung Ho Lim', Kyoung Min Kim²,3 \\ ${ }^{1}$ Department of Psychology, College of Health Science, Dankook University, ${ }^{2}$ Department of Psychiatry, Dankook University Hospital, \\ ${ }^{3}$ Department of Psychiatry, College of Medicine, Dankook University, Cheonan, Korea
}

Objective: Attention-deficit/hyperactivity disorder (ADHD) and tic disorder (TD) are among the most common comorbid psychopathologies and have a shared genetic basis. The psychopathological and neurophysiological aspects of the mechanism underlying the comorbidity of both disorders have been investigated, but the pathophysiological aspects remain unclear. Therefore, this study aimed to compare the neurophysiological characteristics of ADHD with those of TD using resting-state electroencephalography and exact low-resolution brain electromagnetic tomography (eLORETA) analysis.

Methods: We performed eLORETA analysis based on the resting-state scalp-recorded electrical potential distribution in 34 children with ADHD and 21 age-matched children with TD. Between-group differences in electroencephalography (EEG) current source density in delta, theta, alpha, beta, and gamma bands were investigated in each cortical region. Results: Compared with the TD group, the ADHD group showed significantly increased theta activity in the frontal region (superior frontal gyrus, $t=3.37, p<0.05$; medial frontal gyrus, $t=3.35, p<0.05$ ). In contrast, children with TD showed decreased posterior alpha activity than those with ADHD (precuneus, $t=-3.40, p<0.05$; posterior cingulate gyrus, $t=-3.38, p<0.05$ ). These findings were only significant when the eyes were closed.

Conclusion: Increased theta activity in the frontal region is a neurophysiological marker that can distinguish ADHD from TD. Also, reduced posterior alpha activity might represent aberrant inhibitory control. Further research needs to confirm these characteristics by simultaneously measuring EEG-functional magnetic resonance imaging.

KEY WORDS: ADHD; Tic disorder; Electroencephalography.

\section{INTRODUCTION}

Attention-deficit/hyperactivity disorder (ADHD) is one of the major neurodevelopmental disorders and is characterized by three symptom domains, namely, hyperactivity, impulsivity, and inattention [1]. The prevalence of $\mathrm{ADHD}$ is reported to be about $5 \%$ in children and $2.5 \%$ in adults, and children with ADHD are commonly encountered in child and adolescent psychiatry clinics $[1,2]$. ADHD is also known to be highly comorbid with other psychiatric disorders, such as anxiety and depression $[3,4]$.

Received: May 20, 2021 / Revised: July 22, 2021

Accepted: July 23, 2021

Address for correspondence: Kyoung Min Kim

Department of Psychiatry, College of medicine, Dankook

University, 119 Dandae-ro, Dongnam-gu, Cheonan 31116, Korea

E-mail: profuture@naver.com

ORCID: https://orcid.org/0000-0003-0577-0701
Tic disorder (TD) is also a common neurodevelopmental disorder, the symptoms of which are sudden, rapid, recurrent, nonrhythmic motor movements or vocalizations [1]. TD includes the diagnostic sub-categories of "provisional tic disorder," "chronic motor or vocal tic disorder," and "Tourette's disorder." Tourette's disorder is a severe form of TD diagnosed when both motor and vocal tics have been present for more than 1 year [1]. Transient tic symptoms are common in the school-aged population, affecting around $15-25 \%$ of children [5]. TD is also known to be highly comorbid with other psychopathologies, including depression, anxiety, disruptive behaviors, and obsessive-compulsive disorder [6].

ADHD and TD are among the most common comorbid psychopathologies and have a shared genetic basis [7]. The prevalence of comorbid ADHD in patients with Tourette's disorder is reported to be about $50 \%$ but ranges

(c) This is an Open-Access article distributed under the terms of the Creative Commons Attribution Non-Commercial License (http://creativecommons.org/licenses/by-nc/4.0) which permits unrestricted non-commercial use, distribution, and reproduction in any medium, provided the original work is properly cited. 
widely from $21-90 \%$ [6,8-10]. Furthermore, $8-14 \%$ of patients with ADHD have been reported to have TD [11]. The psychopathological and neurobiological aspects of the mechanism underlying the comorbidity of these disorders have been investigated by multiple previous studies [12-15]. However, the pathophysiological mechanism of this comorbidity is still unclear.

Over the past two decades, electroencephalography (EEG) studies have been conducted to investigate the neurobiological characteristics of neurodevelopmental disorders [16-18]. The aberrant resting-state EEG can be considered as valuable information in the temporal dynamics of brain function. Previous studies of the association between ADHD and spontaneous EEG activity have generally reported increased slow-wave activity (i.e., delta and theta power) in the frontal area of the brain $[19,20]$. A meta-analysis of nine EEG studies in patients with ADHD and controls found an increase in theta power and a decrease in beta power, resulting in an increased theta/beta power ratio with an effect size of 3.08 in ADHD [19]. Based on these findings, the high theta/beta ratio was suggested as a tool for evaluating ADHD [21], although its use was recommended within the limits of a research study due to its lack of evidence and high false-positive rate $[21,22]$.

The previous studies for the EEG findings in patients with TD were performed in relation to the tics suppression and found increased connectivity between brain regions and increased alpha and beta band oscillations during the suppression of tics [23-26]. For instance, Zapparoli et al. [24] also reported a decreased level of beta power in patients with TD than the healthy controls during the execution of some tasks; however, this abnormal pattern became normal during the voluntary tics suppression. Morand-Beaulieu also investigated the EEG changes during tic suppression and found an increased alpha-band connectivity during tic suppression in children with TD [25]. Meanwhile, other studies using the resting state EEG in patients with TD also found reduced EEG complexity index values (e.g., sample entropy) in all channels and decreased connectivity between the frontal and tempo$\mathrm{ral} /$ occipital/parietal lobes, compared to healthy controls $[27,28]$.

As aforementioned, ADHD and TD are highly comorbid conditions with common genetic components. However, despite the multiple studies for the EEG findings of each psychopathology comparing with the normal controls, respectively, there is still a lack of studies that directly compared the resting-state EEG between both psychopathologies of ADHD and TD. Moreover, studies considering the volume conduction effect are also scarce. This is the important factor of spectrum analysis EEG at sensor level that may cause lower reliability or type 1 error [29]. Exact low-resolution brain electromagnetic tomography (eLORETA) is a three-dimensional visualization tool that reconstructs cortical electrical activity from EEG data with correct localization [29]. Using the principles of linearity and superposition, eLORETA produces a low-resolution estimate of any distribution of electrical neuronal activity [30]. The usefulness and accuracy of eLORETA in solving the inverse problem of different brain disorders with EEG data have been demonstrated $[20,31,32]$. Thus, the present study aimed to compare the neurophysiological characteristics between these two developmental disorders using resting-state EEG with eLORETA analysis.

\section{METHODS}

\section{Participants}

A total of 55 children and adolescents, who visited the outpatient clinic of child and psychiatry in a university hospital, Republic of Korea, were recruited. The exclusion criteria were: (1) no previous history of a head injury, somatic disorder, or epilepsy; (2) no loss of consciousness; and (3) no diagnosis of intellectual disability based on the Diagnostic and Statistical Manual of Mental Disorders 5th edition. Thirty-four patients diagnosed with ADHD (24 boys, 10 girls; mean age $10.32 \pm 2.34$ years) and 21 patients with TD (19 boys, 2 girls; mean age 9.76 \pm 2.23 years) matched for age and full-scale intelligence quotient were recruited. All subjects were right-handed. Some of the study participants were receiving medication and were asked not to take it for at least 72 hours before the experiment. The parent(s) of each child provided written informed consent after children and parents received an explanation of the study purpose and methodology. The study protocol was approved by the ethical review committee of Dankook University Hospital (IRB No. 2020-06-013). 


\section{Clinical Assessments}

\section{Intelligence test}

To evaluate the participants' intelligence, we used the standardized version of the Korean-Wechsler Intelligence Scale for Children-Fourth Edition [33,34], which is designed to evaluate the cognitive ability of children and adolescents aged $6-16$ years.

\section{Behavioral assessment}

Participants' behavioral outcomes were assessed using the Child Behavior Checklist (CBCL), a multi-faceted assessment scale to assess emotional and behavioral problems in children and adolescents [35]. It contains 119 questions, and each question is answered as "does not correspond to this at all" (0 points), "sometimes or so" (1 point), and "often or very often" (2 points). The CBCL includes eight subscales, including withdrawal, physical symptoms, depression/anxiety, social problems, thinking problems, attention problems, delinquency, and aggression, as well as an internalization problem scale, externalization problem scale, and total problem behavior scale.

\section{Korean version of the Dupaul ADHD rating scale}

The severity of ADHD features was assessed using the Korean version of the Dupaul ADHD rating scale (K-ARS), which consists of 18 questions, each of which is scored on a 4-point scale of $0-3$. This scale includes nine items about attention-deficit and nine questions about hyperactivity/impulsivity [36]. Odd-numbered questions evaluate carelessness, and even-numbered questions evaluate hyperactivity/impulsivity. The standardization of K-ARS has been established [37].

\section{Yale Tic Symptom Rating Scale}

The severity of TD was evaluated using the Yale Global Tic Symptom Rating Scale (YGT-SS). This scale was developed by Leckman et al. [38] in 1989 and allows skilled clinicians to assess the severity of tic symptoms by semi-structured interviews with various information providers. The YGT-SS includes a self-reported questionnaire and evaluation by direct observation. First, the clinician asks the patient's family about the pattern and body distribution of tic symptoms in the previous week, administers the patient semi-structured interview, and then records a list of tic symptoms. After completing the semi-structured interview using a list of tic symptoms as a guide, the clinician evaluated motor and vocal tics in five dimensions, namely, number, frequency, intensity, complexity, and interference of motor and vocal tics over the past weeks. For each dimension, a 6-point scale ranging from 0 to 5 for each domain is used with a separate item for the overall impairment regarding the subject's daily life.

\section{EEG Recordings and Data Acquisition}

An EEG was recorded for each patient using a digital BioBrain-32 dried-cap device (BioBrain Inc., Daejeon, Korea) with the electrodes positioned according to the International 10-20 system (i.e., Fp1, Fp2, AF3, AF4, F3, Fz, F4, F7, F8, FC3, FCz, FC4, C3, Cz, C4, FT7, FT8, T7, T8, TP7, TP8, CP3, CPz, CP4, P3, Pz, P4, P7, P8, O1, Oz, and $\mathrm{O} 2$ ). The EEG activity was acquired using $\mathrm{FCz}$ as the reference and $\mathrm{FPz}$ as the ground with a sampling rate of $250 \mathrm{~Hz}$. Vertical eye movement was recorded from two electrodes placed above and below the left eye, with horizontal eye movements recorded from electrodes placed on the outer canthus of each eye. Online filtering was performed between 1 and $50 \mathrm{~Hz}$ and notch filtering at $60 \mathrm{~Hz}$. Impedance was kept below $50 \mathrm{k} \Omega$ because of the dry electrodes used with the EEG acquisition cap.

\section{Collection and Preprocessing of Resting-state EEG Data}

The experiment was performed in a noise-attenuated room. Resting-state EEG data were recorded for 3 minutes each with the eyes open and closed on two occasions (i.e., EO-EC-EO-EC, total 12 minutes). The participants were awake, comfortably seated, and requested to relax and try not to move. All data were exported in ASCII format. EEG preprocessing was performed with MATLAB R2015a (MathWorks, Natick, MA, USA) and used EEGLAB v14.0. The raw data were sampled to $250 \mathrm{~Hz}$ and re-referenced to the average. The EEG data were filtered with a $1-50 \mathrm{~Hz}$-bandpass filter. Off-line artifact rejection was then performed by visual inspection to eliminate epochs caused by body movement or a bad channel. Finally, independent component analysis was performed to eliminate ocular and prominent muscle artifacts [39], including tic movements. 


\section{EEG Source Localization and Statistical Analysis}

eLORETA was used to compute the intracortical distribution of the electrical activity from the surface EEG data [29]. This method is a discrete, three-dimensionally distributed, linear, weighted minimum norm inverse solution. The weights used in eLORETA provide tomography with the property of exact localization to test point sources, yielding images of current density with exact localization, albeit with a low spatial resolution (i.e., neighboring neuronal sources are highly correlated). A further property of eLORETA is that it has no localization bias even in the presence of structured noise [30].

In this study, the solution space was restricted to the cortical gray matter, corresponding to 6239 voxels at a spatial resolution of $5 \times 5 \times 5 \mathrm{~mm}$. The Montreal Neurological Institute average MRI brain (MNI152) was used as a realistic head model, for which the lead field was computed $[40,41]$. The validity of eLORETA tomography was confirmed in previous validation studies of LORETA and sLORETA $[42,43]$.

Selected artifact-free EEG segments were used to calculate the eLORETA intracranial spectral density from 1-50 $\mathrm{Hz}$ with a resolution of $1 \mathrm{~Hz}$. Functional eLORETA images of spectral density were computed for seven frequency bands: delta $(1-4 \mathrm{~Hz})$, theta $(4-8 \mathrm{~Hz})$, alpha $(8-12 \mathrm{~Hz})$, beta1 $(13-21 \mathrm{~Hz})$, beta2 $(21-30 \mathrm{~Hz})$, beta3 $(30-40$ $\mathrm{Hz})$, and gamma $(40-50 \mathrm{~Hz})$.

The difference in cortical oscillations in each frequency band and the localized source between groups was assessed voxel-by-voxel by the independent-samples $t$ test and the paired $t$ test based on eLORETA log-transformed current density power. In the resulting statistical three-dimensional images, cortical voxels showing significant differences were identified by a nonparametric approach (statistical nonparametric mapping) via randomizations [44]. In the resulting three-dimensional statistical mapping, cortical voxels with significant differences were identified by means of a nonparametric permutation and randomization procedure with the threshold set at the 5\% probability level, comparing the mean source power in each voxel and the distribution in the permutated values. This randomization strategy determined the critical probability threshold values for the observed $t$ values with correction for multiple comparisons across all voxels and all frequencies. The correction is exact for a large number of randomizations, based on Fisher's permutation method. Thus, the results do not need to rely on Gaussianity [44]. By evaluating the empirical probability distribution of the "maximal statistics" in the null hypothesis, permutation and randomization tests have been demonstrated to effec-

Table 1. Demographic and clinical characteristics of participants

\begin{tabular}{|c|c|c|c|c|}
\hline Variables & $\mathrm{ADHD}(\mathrm{n}=34)$ & Tic disorder $(n=21)$ & $d f$ & $\chi^{2}$ or $t$ \\
\hline Age & $10.32 \pm 2.34$ & $9.76 \pm 2.23$ & 53 & -0.918 \\
\hline Sex, female/male (n) & $10 / 24$ & $2 / 19$ & & 0.083 \\
\hline Full Scale IQ & $82.62 \pm 9.62$ & $84.19 \pm 6.94$ & 53 & 0.878 \\
\hline YGT-SS total score & $2.97 \pm 4.71$ & $25.33 \pm 15.5$ & 22.29 & $-6.40^{* * *}$ \\
\hline K-ARS total score & $26.88 \pm 4.68$ & $14.62 \pm 4.99$ & 53 & $9.20^{* * *}$ \\
\hline \multicolumn{5}{|l|}{ CBCL } \\
\hline Total problem score & $70.59 \pm 10.54$ & $61.67 \pm 7.63$ & 53 & $3.35^{* * *}$ \\
\hline Internalizing & $62.62 \pm 10.83$ & $56.81 \pm 6.76$ & 53 & $2.202^{*}$ \\
\hline Externalizing & $67.91 \pm 11.21$ & $60.48 \pm 7.95$ & 53 & $2.65^{*}$ \\
\hline Anxious/depressed & $63.18 \pm 9.26$ & $57.24 \pm 6.97$ & 53 & $2.526^{*}$ \\
\hline Withdrawn & $59.85 \pm 9.28$ & $57.1 \pm 6.72$ & 53 & 1.181 \\
\hline Somatic complain & $58.38 \pm 8.38$ & $55.95 \pm 4.88$ & 53 & 1.205 \\
\hline Social problem & $69.06 \pm 8.46$ & $61.24 \pm 7.19$ & 53 & $3.518^{* * *}$ \\
\hline Thought problem & $63.59 \pm 7.72$ & $62 \pm 5.53$ & 53 & 0.82 \\
\hline Attention problem & $68.68 \pm 8.86$ & $62.43 \pm 7.06$ & 53 & $2.735^{* *}$ \\
\hline Delinquent behavior & $62.35 \pm 7.21$ & $57.71 \pm 6.81$ & 53 & $2.366^{*}$ \\
\hline Aggressive behavior & $66.35 \pm 9.06$ & $61.48 \pm 6.12$ & 53 & $2.173^{*}$ \\
\hline
\end{tabular}

Values are presented as mean \pm standard deviation.

ADHD, attention-deficit/hyperactivity disorder; IQ, intelligent quotient; YGT-SS, Yale Global Tic Severity Scale; K-ARS, Korean version of ADHD rating scale; $\mathrm{CBCL}$, Child Behavior Checklist.

${ }^{*} p<0.05 ; * *<0.01 ; * * * p<0.001$. 
tively control type I error in neuroimaging studies [44]. Demographic and clinical data were examined using the independent $t$-test with SPSS version 26 (IBM Corp., Armonk, NY, USA).

\section{RESULTS}

\section{Demographic and Clinical Characteristics}

The patient demographics and clinical characteristics are shown in Table 1 . There was no significant between-group difference in the distributions of patient age (8-12 years), sex, and the full-scale intelligence quotient. The CBCL and K-ARS scores were significantly higher in patients with ADHD than in those with TD (CBCL total problem, $t=3.35, p<0.001$; K-ARS, $t=9.20, p<0.001$ ) while YGT-SS scores were significantly higher in patients with TD (YGT-SS total, $t=-6.40, p<0.001$ ).

\section{Averaged Current Source Density for each Frequency Band in ADHD and TD}

Figure 1 shows the averaged eLORETA solutions for each frequency band in the resting-state condition in both study groups. The highest current source density (CSD) values were found in the alpha band in the ADHD group $(\mathrm{EC}$ alpha $=5.34 ; \mathrm{EO}$ alpha $=3.96)$. There was a similar slow-wave CSD (Theta band) in both the EO and EC conditions of the ADHD group over the frontal cortex. On the other hand, alpha cortical sources showed high CSD over the parieto-occipital regions in the ADHD group. The statistical analysis did not reveal any significant difference in every frequency band between $\mathrm{EO}$ and $\mathrm{EC}$ conditions of the ADHD group. Contrary to the ADHD group, the children with TD showed significant differences in alpha and beta band activities between EO and EC conditions.

\section{Comparison of Source Localization between the ADHD and TD Group in EC Condition}

Figure 2 shows the statistical differences of CSD between children with ADHD and TD in EC condition, with Figure $2 \mathrm{~A}$ representing the differences of theta activity and Figure $2 \mathrm{~B}$ representing the differences of alpha activity. Source localization analysis showed significantly higher CSD values for theta band activity in the ADHD group, compared to children with TD. over the frontal region (max $t$ threshold: 3.34 for $p<0.05$; superior frontal gyrus, $t=3.37$; medial frontal gyrus, $t=3.35$; corrected for multi-
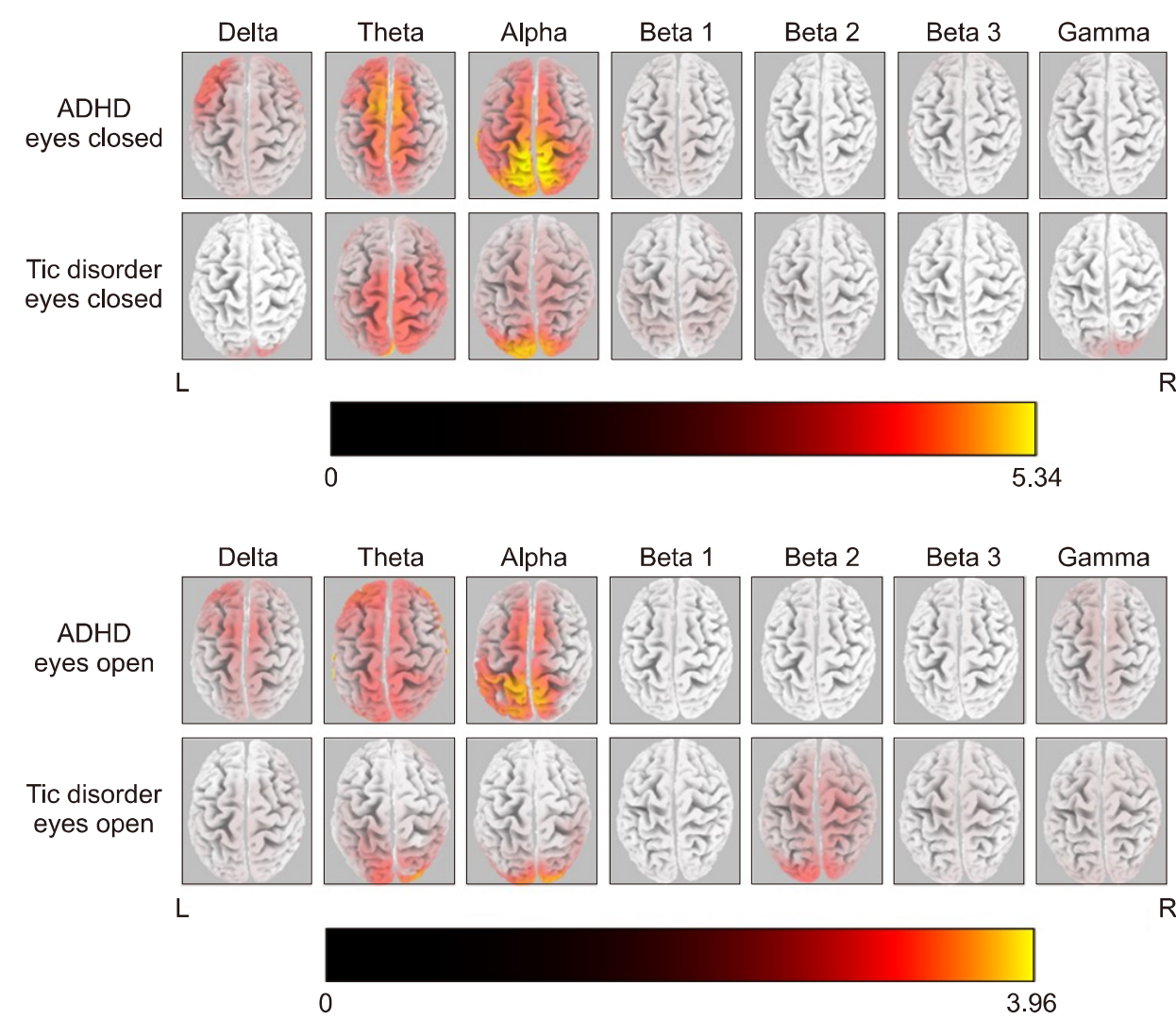

Fig. 1. Averaged eLORETA current source density of each frequency band in $\mathrm{EO}$ and $\mathrm{EC}$ conditions of both groups of ADHD and TD. A current density value from each frequency band was calculated and fixed with maximum global power (max CSD fixed with alpha band value for $\mathrm{EC}=5.34$; $\max \mathrm{CSD}$ fixed with alpha band value for $\mathrm{EO}=$ 3.96).

ADHD, attention-deficit/hyperactivity disorder; TD, tic disorder; CSD, current source density; EO, eye-open; EC, eye-closed; eLORETA, exact lowresolution brain electromagnetic tomography; L, left; R, right. 

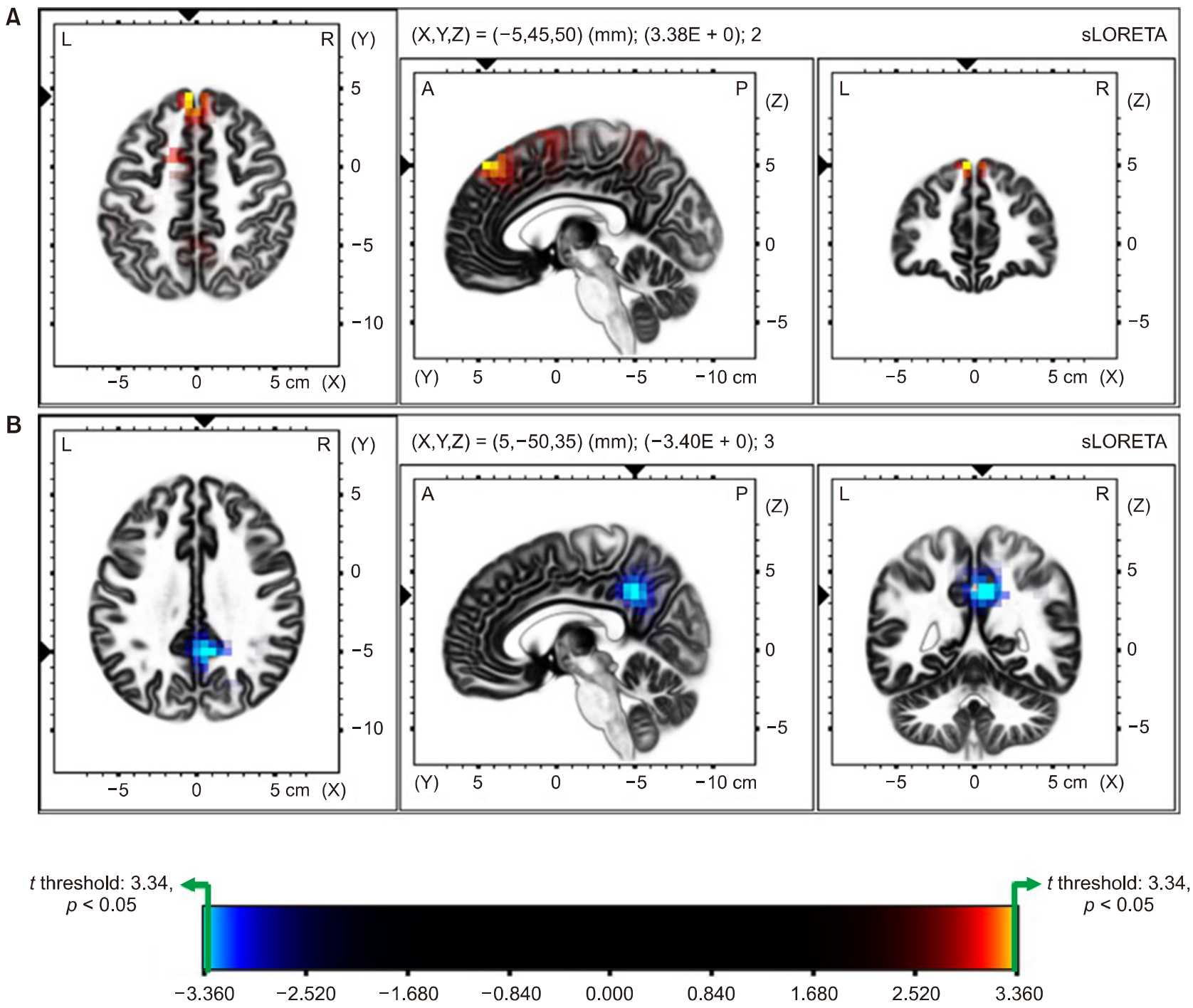

Fig. 2. Comparison of Source Localization between the ADHD and TD group in EC condition. Colored areas represent the spatial extent of voxels with a significant difference between ADHD and TD groups (yellow and cyan blue-coded for $p<0.05$, corrected for multiple comparisons) in current source density. Significant results are projected onto a brain MRI template. The MRI slices are located at the MNI-space coordinates indicated in the figure that corresponds to the voxel of the highest significance. The color scale represents $t$ values $(\max t$ threshold $= \pm 3.34, p<0.05$ ). (A) Represents the differences of theta activity between the groups. ADHD shows increased theta activity than TD, mainly in the frontal lobe (superior frontal gyrus, $t=3.38, p<0.05$; medial frontal gyrus, $t=3.35, p<0.05$ ). (B) Represents the differences of alpha activity. TD group showed decreased alpha activity than ADHD in EC condition, mainly posterior region (precuneus, $t=-3.40, p<0.05$; posterior cingulate gyrus, $t=-3.38$, $p<0.05)$.

ADHD, attention-deficit/hyperactivity disorder; TD, tic disorder; CSD, current source density; EO, eye-open; EC, eye-closed; eLORETA, exact low-resolution brain electromagnetic tomography; MNI, Montreal Neurological Institute; MRI, magnetic resonance imaging.

ple comparison with nonparametric randomization; Fig. $2 \mathrm{~A})$.

On the other hand, current densities for alpha band activity were significantly decreased in TD group than the ADHD group, over the parietal region $\left(_{\max } t\right.$ threshold: -3.34 , for $p<0.05$; precuneus, $t=-3.40$; posterior cingulate gyrus, $t=-3.38$; corrected for multiple comparison with nonparametric randomization; Fig. 2B).
Unlike the EC condition, there was no significant differences of EEG CSD between ADHD and TD in EO condition (one-tailed [A $>\mathrm{B}]_{\max } t$ threshold: 3.46, $p=0.26$; one-tailed $[\mathrm{A}<\mathrm{B}]_{\max } t$ threshold: $-3.52, p=0.17$ ).

\section{Comparison of Source Localization between the EO and EC Conditions in the ADHD Group}

There was no significant difference in CSD for every 
EEG frequency band between $\mathrm{EO}$ and $\mathrm{EC}$ conditions in the ADHD group (one-tailed [A $>$ B] max $t$ threshold: 2.49, $p=0.26$; one tailed $[\mathrm{A}<\mathrm{B}]_{\max } t$ threshold: $-3.11, p=$ 0.09).

\section{Comparison of Source Localization between the EO and EC Conditions in the TD Group}

Statistical nonparametric mapping identified significant differences of EEG activities between the EO and EC conditions in the TD group (Fig. 3). Source localization analysis showed higher CSD values for alpha-band activity in EC condition than the EO condition, which was statistically significant in the posterior region, namely, the posterior cingulate (Fig. 3A; max $t$ threshold: 4.62 for $p<0.05$; posterior cingulate, $t=4.88$; corrected for multiple comparison with nonparametric randomization). Furthermore,

A

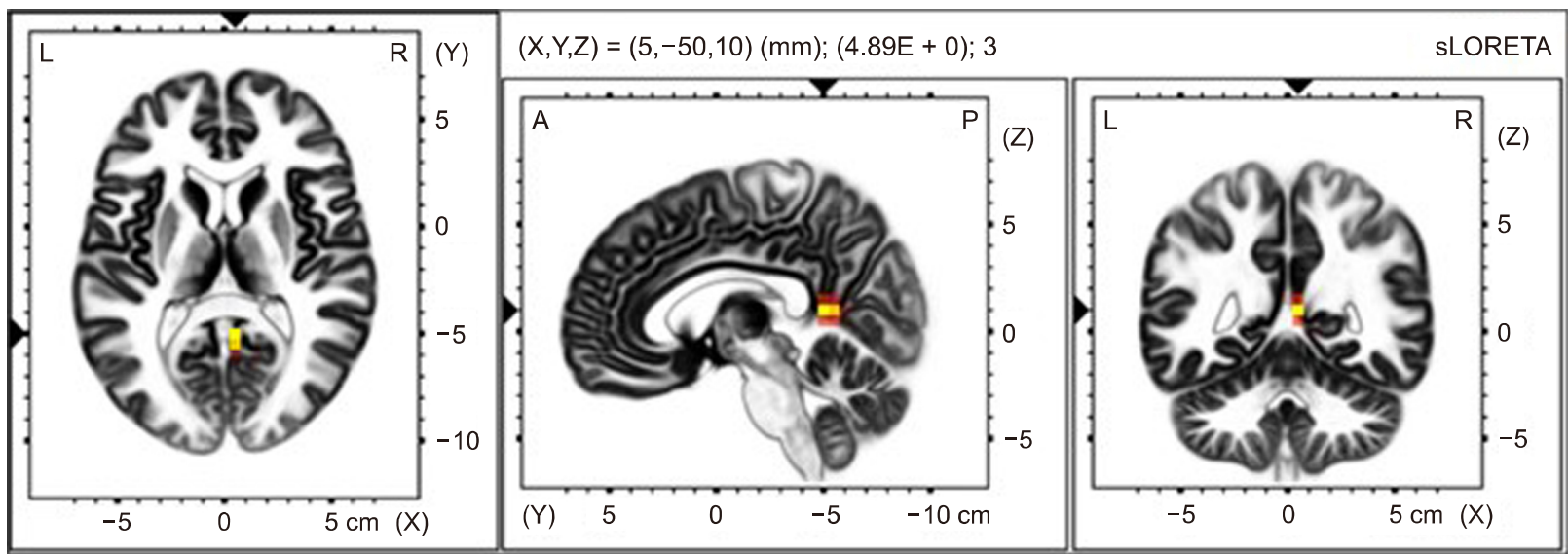

B

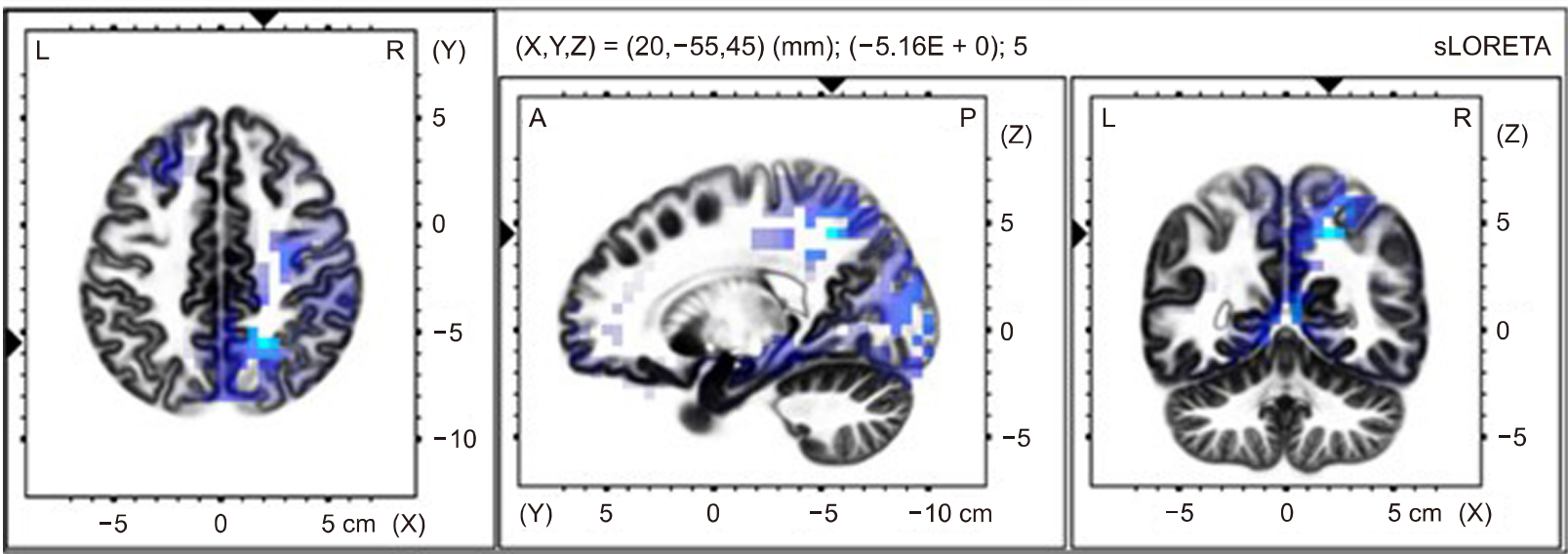

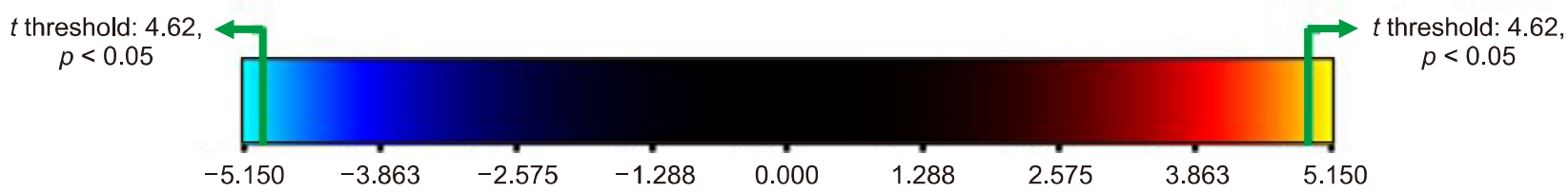

Fig. 3. Comparison of Source Localization between the EO and EC conditions in the TD Group. Colored areas represent the spatial extent of voxels with a significant difference (yellow and cyan blue-coded for $p<0.05$, corrected for multiple comparisons) in current source density between EC and EO in TD. (A) Shows the differences of alpha activity and (B) shows the differences of beta activity, respectively. Significant results are projected onto a brain MRI template. The MRI slices are located at the MNI-space coordinates indicated in the figure that corresponds to the voxel of the highest significance. The color scale represents $t$ values $(\max t$ threshold $= \pm 4.62, p<0.05)$. (A) EC condition shows the significantly increased alpha activity in TD over the parietal lobe. (B) EO condition shows the significantly increased beta activity than EC in TD, over the posterior and occipital region.

TD, tic disorder; EO, eye-open; EC, eye-closed; eLORETA, exact low-resolution brain electromagnetic tomography; MNI, Montreal Neurological Institute; MRI, magnetic resonance imaging. 
CSD values for beta-band activity in TD group for $\mathrm{EO}$ condition were significantly higher than the EC condition in the parietal and occipital regions, namely the precuneus, posterior cingulate, superior parietal lobule, and cuneus (Fig. $3 \mathrm{~B}$; max $t$ threshold: -4.67 for $p<0.05$; precuneus, $t=-5.15$; posterior cingulate, $t=-5.08$; cuneus, $t=$ -4.94; and superior parietal lobule, $t=-4.82$; corrected for multiple comparison with nonparametric randomization).

\section{DISCUSSION}

The present study investigated the neurophysiological differences in the resting state EEG between the age- and intelligence-matched children with ADHD and TD by analyzing CSD detected with eLORETA. Previous resting-state EEG studies in ADHD have shown that spectral power or CSD is higher in the theta band and lower in the alpha and/or beta bands in children with ADHD than in healthy controls [20,45-49]. A recent review of 65 studies for the resting-state EEG in children with ADHD found very high consistency and validation scores in the increased relative power of the slow-wave (e.g., delta and theta bands) in the EC condition [50]. Elevated theta power in the resting state has been considered a sign of cortical slowing (i.e., hypo-arousal), contributing to the inattentional symptoms in children with ADHD [19]. Our findings of increased theta CSD in children with ADHD are consistent with the previous studies, even when compared to TD.

Previous studies reported that the theta activities in the mid frontal area reflect a neural mechanism of cognitive control associated with the attention process [51-53]. They are also associated with working memory, and an increase in theta power was related to the amount of task loading $[54,55]$. Theta activities also predicted lower response time variability at the trial level in ADHD children than healthy controls [56]. Furthermore, regarding age-dependent properties in EEG, our results are partly in agreement with the findings reported by Clarke et al. [49] and Ahmadi et al. [20], who noted a general increase in frontal theta power in 8-12-year-old children with ADHD compared with healthy controls. Overall, the increased theta activity in the frontal region might reflect their pathophysiological characteristic due to an immaturity of development in the brain.
Moreover, our within-group analysis of children with ADHD did not find a statistically significant difference in alpha CSD in two resting conditions (i.e., between the EO and EC conditions), which indicates that alpha CSD does not decrease in the resting state when the eyes are open. It is well known that alpha activity in the posterior region is dominant in normal individuals when the eyes are closed in the resting state and that this activity is suppressed by visual stimulation [57]. This "alpha block" pattern represents a state of arousal, and alpha oscillations functionally inhibit specific regions, which serves to route information by blocking task-irrelevant pathways [58,59]. Several studies reported that posterior alpha desynchronization is associated with visual working memory, visual attention, and state of arousal in healthy subjects $[48,60]$. In our study, alpha desynchronization was not observed in children with ADHD in the EO condition, which implicates the maintenance of hypo-arousal status that might be associated with inattentiveness.

Although TD is one of the major neurodevelopmental disorders, there is a paucity of research on EEG activity during the resting state in children with TD. Weng et al. [27] compared the resting-state EEG between the children with TD and healthy controls and found that the EEG complexity index values (e.g., sample entropy) in all channels were reduced in children with TD, serving as a marker of disturbed brain connectivity. The present study found that children with TD showed attenuated alpha activity in the posterior region (i.e., the precuneus and posterior cingulate gyrus) when compared with their counterparts with ADHD in the EC condition. Thus, our findings for the attenuated alpha activity in TD could be considered in association with the impairment of the inhibitory process, despite the paucity of previous studies.

The spontaneous alpha oscillation is the most ubiquitous cortical electroencephalographic oscillation at rest, and the properties of alpha power have been considered as one of the neurophysiological biomarkers of cortical excitation or inhibition [61-63]. In the framework of sensory aspects of TD (e.g., Tourette's syndrome), researchers have suggested that the symptoms of TD are related with sensory features, characterized by the premonitory urge, which represents the hypersensitivity of patients with TD [64]. Previous studies have reported that the attenuated alpha power was associated with decreased inhibitory sensory processing [61-63]. Regarding 
alpha oscillation, as inhibitory oscillation, our result might reflect the aberrant interoceptive sensory process in children with TD [65]. Previous studies in adults with TD demonstrated that alpha coherence between the prefrontal and cortical motor areas increased when the participants were engaged in voluntary suppression of their tics $[23,66]$. Furthermore, Serrien et al. [23] compared event-related EEG data between the TD group and healthy controls and reported that EEG alpha coherence in the fronto-mesial network increased significantly more in the TD group when they suppressed voluntary movements during the Go/NoGo task, compared to controls. These findings might be explained by the positive association between the increase in alpha activity and behavioral inhibition. Functional MRI studies in children with TD also reported the decreased activity in the posterior cingulate gyrus and precuneus area, suggesting a disrupted cortical control circuit that likely causes failure of inhibition of tic behaviors in children with TD $[67,68]$. These could be considered consistent with our finding of decreased alpha activity in the same brain region.

\section{Limitations}

The present has some limitations that should be noted. First, it did not include a healthy control group. Further studies that include age-matched controls are needed to confirm the present findings. Second, although channel-specific EEG and voxel-specific analysis were performed, brain interactions between regions were not investigated. EEG-based network analysis reflects the effect on temporal dynamics between brain regions, and further study, including the network analysis, is needed to provide additional evidence for the EEG characteristics in children with ADHD and TD.

This is the first study that compared the neurophysiological characteristics of ADHD and TD in children using the resting-state EEG with eLORETA. The children with ADHD presented significantly higher theta activity in the frontal area than the children with TD. On the other hand, the children with TD showed significantly decreased alpha activity in the precuneus and posterior cingulate gyrus than children with ADHD. This finding may represent a decrease in the inhibitory process in the posterior region of the brain in children with TD.

\section{Acknowledgments}

This research was funded by the Institute of Information \& Communications Technology Planning \& Evaluation (IITP), Ministry of Science and ICT, Republic of Korea (ID: 2020-0-00506).

\section{Conflicts of Interest}

No potential conflict of interest relevant to this article was reported.

\section{Author Contributions}

Conceptualization: Ilju Lee, Jiryun Lee, Myung Ho Lim, Kyoung Min Kim. Protocol development: Ilju Lee, Jiryun Lee, Kyoung Min Kim. Funding acquisition: Kyoung Min Kim. Data acquisition: Jiryun Lee, Kyoung Min Kim. Data analysis: Ilju Lee, Kyoung Min Kim. Draft writing: Ilju Lee, Kyoung Min Kim. Writing-review \& editing: Ilju Lee, Jiryun Lee, Myung Ho Lim, Kyoung Min Kim.

\section{ORCID}

Ilju Lee https://orcid.org/0000-0003-3735-3179

Jiryun Lee Myung Ho Lim https://orcid.org/0000-0002-9170-9140

Kyoung Min Kim https://orcid.org/0000-0003-0577-0701

\section{REFERENCES}

1. American Psychiatric Association. Diagnostic and statistical manual of mental disorders (DSM-5 ${ }^{\circledR}$. 5th ed. Washington, DC:American Psychiatric Publishing;2013.

2. Torun $\mathrm{YT}$, Güney E, Aral A, Büyüktaşkin D, Tunca H, Taner YI, et al. Determination of serum vascular endothelial growth factor levels in attention deficit hyperactivity disorder: a case control study. Clin Psychopharmacol Neurosci 2019;17:517522.

3. Schatz DB, Rostain AL. ADHD with comorbid anxiety: a review of the current literature. J Atten Disord 2006;10:141 149.

4. Daviss WB. A review of co-morbid depression in pediatric ADHD: etiology, phenomenology, and treatment. I Child Adolesc Psychopharmacol 2008; 18:565-571.

5. Cohen SC, Leckman JF, Bloch MH. Clinical assessment of Tourette syndrome and tic disorders. Neurosci Biobehav Rev 2013;37:997-1007.

6. Hirschtritt ME, Lee PC, Pauls DL, Dion Y, Grados MA, Illmann $\mathrm{C}$, et al. Lifetime prevalence, age of risk, and genetic relationships of comorbid psychiatric disorders in Tourette syndrome. JAMA Psychiatry 2015;72:325-333. 
7. Tsetsos F, Padmanabhuni SS, Alexander J, Karagiannidis I, Tsifintaris M, Topaloudi A, et al. Meta-analysis of Tourette syndrome and Attention Deficit Hyperactivity Disorder provides support for a shared genetic basis. Front Neurosci 2016; 10:340.

8. Robertson MM. A personal 35 year perspective on Gilles de la Tourette syndrome: prevalence, phenomenology, comorbidities, and coexistent psychopathologies. Lancet Psychiatry 2015;2:68-87.

9. Eapen V, Robertson MM. Are there distinct subtypes in Tourette syndrome? Pure-Tourette syndrome versus Tourette syndrome-plus, and simple versus complex tics. Neuropsychiatr Dis Treat 2015;11:1431-1436.

10. Robertson MM. Attention deficit hyperactivity disorder, tics and Tourette's syndrome: the relationship and treatment implications. A commentary. Eur Child Adolesc Psychiatry 2006;15:1-11.

11. Biederman J, Kwon A, Aleardi M, Chouinard VA, Marino T, Cole $\mathrm{H}$, et al. Absence of gender effects on attention deficit hy peractivity disorder: findings in nonreferred subjects. Am J Psychiatry 2005;162:1083-1089.

12. Roessner V, Becker A, Banaschewski T, Rothenberger A. Executive functions in children with chronic tic disorders with/without ADHD: new insights. Eur Child Adolesc Psychiatry 2007;16 Supp/ 1:36-44.

13. Roessner V, Becker A, Banaschewski T, Rothenberger A. Psychopathological profile in children with chronic tic disorder and co-existing ADHD: additive effects. J Abnorm Child Psychol 2007;35:79-85.

14. Rothenberger A, Banaschewski $\mathrm{T}$, Heinrich $\mathrm{H}, \mathrm{Moll} \mathrm{GH}$, Schmidt $\mathrm{MH}$, van't Klooster B. Comorbidity in ADHD-children: effects of coexisting conduct disorder or tic disorder on event-related brain potentials in an auditory selective-attention task. Eur Arch Psychiatry Clin Neurosci 2000;250:101-110.

15. Yordanova J, Dumais-Huber C, Rothenberger A, Woerner W. Frontocortical activity in children with comorbidity of tic disorder and attention-deficit hyperactivity disorder. Biol Psychiatry 1997:41:585-594.

16. Chi MH, Chu CL, Lee IH, Hsieh YT, Chen KC, Chen PS, et al. Altered auditory $\mathrm{P} 300$ performance in parents with attention deficit hyperactivity disorder offspring. Clin Psychopharmacol Neurosci 2019;17:509-516.

17. Lee YJ, Jeong MY, Kim JH, Kim JS. Associations between the mismatch-negativity potential and symptom severity in medication-naïve children and adolescents with symptoms of attention deficit/hyperactivity disorder. Clin Psychopharmacol Neurosci 2020;18:249-260.

18. Clarke AR, Barry RJ, Johnstone S. Resting state EEG power research in Attention-Deficit/Hyperactivity Disorder: a review update. Clin Neurophysiol 2020;131:1463-1479.

19. Snyder SM, Hall JR. A meta-analysis of quantitative EEG power associated with attention-deficit hyperactivity disorder. J Clin Neurophysiol 2006;23:440-455.
20. Ahmadi M, Kazemi K, Kuc K, Cybulska-Klosowicz A, Zakrzewska M, Racicka-Pawlukiewicz E, et al. Cortical source analysis of resting state EEG data in children with attention deficit hyperactivity disorder. Clin Neurophysiol 2020;131:2115-2130.

21. Nuwer MR, Buchhalter J, Shepard KM. Quantitative EEG in attention-deficit/hyperactivity disorder: a companion payment policy review for clinicians and payers. Neurol Clin Pract 2016;6:543-548.

22. Ogrim G, Kropotov J, Hestad K. The quantitative EEG theta/beta ratio in attention deficit/hyperactivity disorder and normal controls: sensitivity, specificity, and behavioral correlates. Psychiatry Res 2012;198:482-488.

23. Serrien DJ, Orth M, Evans AH, Lees AJ, Brown P. Motor in hibition in patients with Gilles de la Tourette syndrome: functional activation patterns as revealed by EEG coherence. Brain 2005; 128(Pt 1):116-125.

24. Zapparoli L, Macerollo A, Joyce EM, Martino D, Kilner JM. Voluntary tic suppression and the normalization of motor cortical beta power in Gilles de la Tourette syndrome: an EEG study. Eur J Neurosci 2019;50:3944-3957.

25. Morand-Beaulieu S, Wu J, Mayes LC, Grantz H, Leckman JF, Crowley MJ, et al. Increased alpha-band connectivity during tic suppression in children with Tourette syndrome revealed by source Electroencephalography analyses. Biol Psychiatry Cogn Neurosci Neuroimaging 2021 doi: 10.1016/j.bpsc. 2021.05.001. [Epub ahead of print]

26. Hong HJ, Sohn H, Cha M, Kim S, Oh J, Chu MK, et al. Increased frontomotor oscillations during tic suppression in children with Tourette syndrome. J Child Neurol 2013;28: 615-624.

27. Weng WC, Chang CF, Wong LC, Lin JH, Lee WT, Shieh JS. Altered resting-state EEG complexity in children with Tourette syndrome: a preliminary study. Neuropsychology 2017;31: 395-402.

28. Duan K, Wu Q, Liao Y, Si Y, Bore JC, Li F, et al. Discrimination of Tourette syndrome based on the spatial patterns of the resting-state EEG network. Brain Topogr 2021,34:78-87.

29. Pascual-Marqui RD, Lehmann D, Koukkou M, Kochi K, Anderer $\mathrm{P}$, Saletu B, et al. Assessing interactions in the brain with exact low-resolution electromagnetic tomography. Philos Trans A Math Phys Eng Sci 2011;369:3768-3784.

30. Pascual-Marqui RD, Biscay-Lirio RJ. Interaction patterns of brain activity across space, time and frequency. Part I: methods. arXiv 2011. doi: 10.5167/uzh-57989.

31. Dattola S, La Foresta F. An eLORETA longitudinal analysis of resting state EEG rhythms in Alzheimer's disease. App/ SCi 2020;10:5666.

32. Smith EE, Cavanagh JF, Allen JJB. Intracranial source activity (eLORETA) related to scalp-level asymmetry scores and depression status. Psychophysiology 2018;55:e13019.

33. O'Donnell $\mathrm{L}$. The Wechsler intelligence scale for children-fourth edition. In: Naglieri JA, Goldstein S, editors. Practitioner's guide to assessing intelligence and achievement. New York:John 
Wiley \& Sons;2009. p. 153-190.

34. Kwak KJ, O S, Kim CT. Korean-Wechsler intelligence scale for children. 4th ed. Seoul:Hakjisa;2011.

35. Achenbach TM, Ruffle TM. The Child Behavior Checklist and related forms for assessing behavioral/emotional problems and competencies. Pediatr Rev 2000;21:265-271.

36. DuPaul GJ, Power TJ, Anastopoulos AD, Reid R. ADHD rating scale-IV: checklists, norms, and clinical interpretation. New York:Guilford Press; 1998.

37. So YK, Noh JS, Kim YS, Ko SG, Koh YJ. The reliability and validity of Korean parent and teacher ADHD rating scale. J Korean Neuropsychiatr Assoc 2002;41:283-289.

38. Leckman JF, Riddle MA, Hardin MT, Ort SI, Swartz KL, Stevenson J, et al. The Yale Global Tic Severity Scale: initial testing of a clinician-rated scale of tic severity. I Am Acad Child Adolesc Psychiatry 1989;28:566-573.

39. Jung TP, Makeig S, Humphries C, Lee TW, McKeown MJ, Iragui $\mathrm{V}$, et al. Removing electroencephalographic artifacts by blind source separation. Psychophysiology 2000;37:163-178.

40. Mazziotta J, Toga A, Evans A, Fox P, Lancaster J, Zilles K, et al. A probabilistic atlas and reference system for the human brain: International Consortium for Brain Mapping (ICBM). Philos Trans R Soc Lond B Biol Sci 2001;356:1293-1322.

41. Fuchs M, Kastner J, Wagner M, Hawes S, Ebersole JS. A standardized boundary element method volume conductor model. Clin Neurophysiol 2002;113:702-712.

42. Vitacco D, Brandeis D, Pascual-Marqui R, Martin E. Correspondence of event-related potential tomography and functional magnetic resonance imaging during language processing. Hum Brain Mapp 2002;17:4-12.

43. Mele G, Cavaliere C, Alfano V, Orsini M, Salvatore M, Aiello M. Simultaneous EEG-fMRI for functional neurological assessment. Front Neurol 2019;10:848.

44. Nichols TE, Holmes AP. Nonparametric permutation tests for functional neuroimaging: a primer with examples. Hum Brain Mapp 2002;15:1-25.

45. Barry RJ, Clarke AR, Johnstone SJ, McCarthy R, Selikowitz M. Electroencephalogram theta/beta ratio and arousal in attention-deficit/hyperactivity disorder: evidence of independent processes. Biol Psychiatry 2009;66:398-401.

46. Arns M, Conners CK, Kraemer HC. A decade of EEG theta/beta ratio research in ADHD: a meta-analysis. J Atten Disord 2013; 17:374-383.

47. Kitsune GL, Cheung $\mathrm{CH}$, Brandeis D, Banaschewski T, Asherson P, McLoughlin G, et al. A matter of time: the influence of recording context on EEG spectral power in adolescents and young adults with ADHD. Brain Topogr 2015;28: 580-590.

48. Koehler S, Lauer P, Schreppel T, Jacob C, Heine M, BoreattiHümmer $\mathrm{A}$, et al. Increased EEG power density in alpha and theta bands in adult ADHD patients. J Neural Transm (Vienna) 2009; 116:97-104.

49. Clarke AR, Barry RJ, McCarthy R, Selikowitz M. Age and sex effects in the EEG: differences in two subtypes of attention-deficit/hyperactivity disorder. Clin Neurophysiol 2001;112:815826.

50. Newson JJ, Thiagarajan TC. EEG frequency bands in psychiatric disorders: a review of resting state studies. Front Hum Neurosci 2019;12:521.

51. Cavanagh JF, Frank MJ. Frontal theta as a mechanism for cognitive control. Trends Cogn Sci 2014;18:414-421.

52. Wascher E, Rasch B, Sänger J, Hoffmann S, Schneider D, Rinkenauer $\mathrm{G}$, et al. Frontal theta activity reflects distinct aspects of mental fatigue. Biol Psychol 2014;96:57-65.

53. van Driel J, Ridderinkhof KR, Cohen MX. Not all errors are alike: theta and alpha EEG dynamics relate to differences in error-processing dynamics. J Neurosci 2012;32:16795-16806.

54. Onton J, Delorme A, Makeig S. Frontal midline EEG dynamics during working memory. Neuroimage 2005;27:341-356.

55. Xie Y, Li Y, Duan H, Xu X, Zhang W, Fang P. Theta oscillations and source connectivity during complex audiovisual object encoding in working memory. Front Hum Neurosci 2021;15: 614950.

56. Guo J, Luo X, Li B, Chang Q, Sun L, Song Y. Abnormal modulation of theta oscillations in children with attention-deficithyperactivity disorder. Neuroimage Clin 2020;27:102314.

57. Pfurtscheller G, Stancák A Jr, Neuper C. Event-related synchronization (ERS) in the alpha band--an electrophysiological correlate of cortical idling: a review. Int I Psychophysiol 1996;24:39-46.

58. Thut G, Miniussi C. New insights into rhythmic brain activity from TMS-EEG studies. Trends Cogn Sci 2009;13:182-189.

59. Klimesch W, Sauseng P, Hanslmayr S. EEG alpha oscillations: the inhibition-timing hypothesis. Brain Res Rev 2007;53: 63-88.

60. Vollebregt MA, Zumer JM, Ter Huurne N, Buitelaar JK, Jensen O. Posterior alpha oscillations reflect attentional problems in boys with Attention Deficit Hyperactivity Disorder. Clin Neurophysiol 2016;127:2182-2191.

61. Mathewson KE, Prudhomme C, Fabiani M, Beck DM, Lleras A, Gratton G. Making waves in the stream of consciousness: entraining oscillations in EEG alpha and fluctuations in visual awareness with rhythmic visual stimulation. J Cogn Neurosci 2012;24:2321-2333.

62. Woltering S, Jung J, Liu Z, Tannock R. Resting state EEG oscillatory power differences in ADHD college students and their peers. Behav Brain Funct 2012;8:60.

63. Deiber MP, Hasler R, Colin J, Dayer A, Aubry JM, Baggio S, et al. Linking alpha oscillations, attention and inhibitory control in adult $A D H D$ with EEG neurofeedback. Neuroimage Clin 2020;25:102145.

64. Cox JH, Seri S, Cavanna AE. Sensory aspects of Tourette syndrome. Neurosci Biobehav Rev 2018;88:170-176.

65. Houghton DC, Capriotti MR, Conelea CA, Woods DW. Sensory phenomena in Tourette syndrome: their role in symptom formation and treatment. Curr Dev Disord Rep 2014;1:245-251. 
66. Niccolai V, van Dijk H, Franzkowiak S, Finis J, Südmeyer M, Jonas $\mathrm{M}$, et al. Increased beta rhythm as an indicator of inhibitory mechanisms in tourette syndrome. Mov Disord 2016; 31:384-392.

67. Cui Y, Jin Z, Chen X, He Y, Liang X, Zheng Y. Abnormal baseline brain activity in drug-naïve patients with Tourette syn- drome: a resting-state fMRI study. Front Hum Neurosci 2014; $7: 913$

68. Liu Y, Wang J, Zhang J, Wen H, Zhang Y, Kang H, et al. Altered spontaneous brain activity in children with early Tourette syndrome: a resting-state $\mathrm{fMRI}$ study. Sci Rep 2017;7:4808. 